\title{
Feedback Control of the Lottery System in Theme Park
}

\author{
Noriaki Sakamoto \\ Faculty of Economics, Hosei University, Tokyo, Japan
}

Email address:

nsaka@hosei.ac.jp

\section{To cite this article:}

Noriaki Sakamoto. Feedback Control of the Lottery System in Theme Park. Automation, Control and Intelligent Systems. Vol. 3, No. 5, 2015, pp. 76-80. doi: 10.11648/j.acis.20150305.13

\begin{abstract}
In Tokyo Disney Park, the guests must reserve the seats by a lottery to view some shows. This paper proposes the robust feedback control system to solve the problem of the lottery system. The controlled variable is winning / losing to the guests who draw the lottery, and the control logic is ON/OFF-Type Discrete Variable Structure Controller, to compensate the uncertainty of a simulation to reproduce the lottery. The simulation that input data are made using many real data shows the effectiveness of the proposed method. Next, Neural Network Model predicts the controlled result. If the bad result is predicted, the staff of the lottery system is able to take an effective measure.
\end{abstract}

Keywords: Discrete Variable Structure Control, Lottery System, Theme Park, Uncertainty, Robust Control

\section{Introduction}

In Tokyo Disney Park (Disneyland and DisneySea) [1], the seats of some shows ("Once Upon a Time", "One Man's Dream II", "Big Band Beat") are reserved by a lottery. For example, to view "Once Upon a Time" in the viewing area, the guests are required the free reserved seat tickets. The tickets are distributed by the lottery from Park Opening Time until 30 minutes before the show start time. Each party can attempt the lottery only once for each show a day.

The difficulties of this lottery system are;

Requirement (1):

When the parties draw the lottery, at that moment, they know they are Winner or Loser.

Requirement (2):

The vacant seats must become zero as possible at 30 minutes before the show starts. Because, if all the seats are reserved early, all parties who come later become losers.

Requirement (3):

The guests in a party must be assigned the seats which are in the same line without a crossing the aisle.

The general lottery methods or research are classified roughly into two [2]. One is that it accepts the application of a lottery in a fixed time (or period) and then the lottery is conducted after the deadline $[3,4]$. The other is a lottery machine (for example, it is performed in a shopping mall). We cannot apply the former method to this lottery system, because the guests have to wait for the result. In addition, as the result affects the plan of the day, it gives restrictions to the guest's schedule. We cannot apply the latter lottery machine as well. If each party thinks that the vacant seats become zero early, they try to draw the lottery as fast as possible and it causes congestion. Also, it is unfair for the guests who enter in the evening.

The technique used in Tokyo Disney Park decides winning or losing based on the winning probability that are written in a learning database [5]. We think that the present system has two problems;

Problem (1):

SNS (Twitter, etc.) has come to be used widely, so many guests know that the lottery system has several minutes of alternate winning-time and losing-time (See Figure 1). As a result, the parties who draw the lottery stay in the place where the lottery machines are lining up until the winning-time begins. Their behaviors disturb the flow of the lottery queue and the work of the cast members who deal with the lottery machine trouble, etc.

Problem (2):

The weather in Japan often change suddenly. We think that the database does not have enough the similar situation data. In fact, the lottery is conducted even if there is a rainstorm or typhoon coming. Very few parties attempt the lottery in such a weather, but not all the parties are winners, so there are many vacant seats left when the show starts.

Therefore, we propose that the new lottery system does not use a database to meet Requirement and to solve Problem. We apply a robust feedback control to compensate for the uncertainties of a simulation data. As one of the robust 
controllers, we use ON/OFF-Type Discrete Variable Structure Controller $[6,7,8]$.

However, this system doesn't solve Problem (2), in addition, we propose that our new lottery system has NN (Neural Network Model) learning all simulation results. NN estimates the number of final vacant seats by using a morning data. The output can warn the lottery staff of a possibility that many seats are remained to be vacant at the show time.

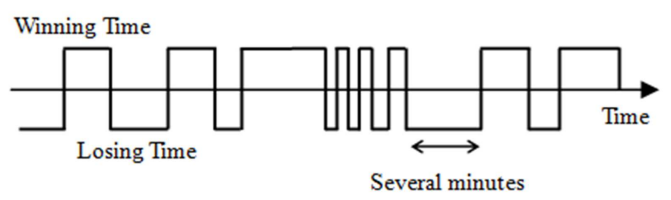

Figure 1. Several minutes of alternate winning-time and losing-time.

\section{Simulation System}

We simulate the lottery system for "Once Upon a Time" by the control block diagram which is shown in Figure 2. Table 1 is a list of symbols.

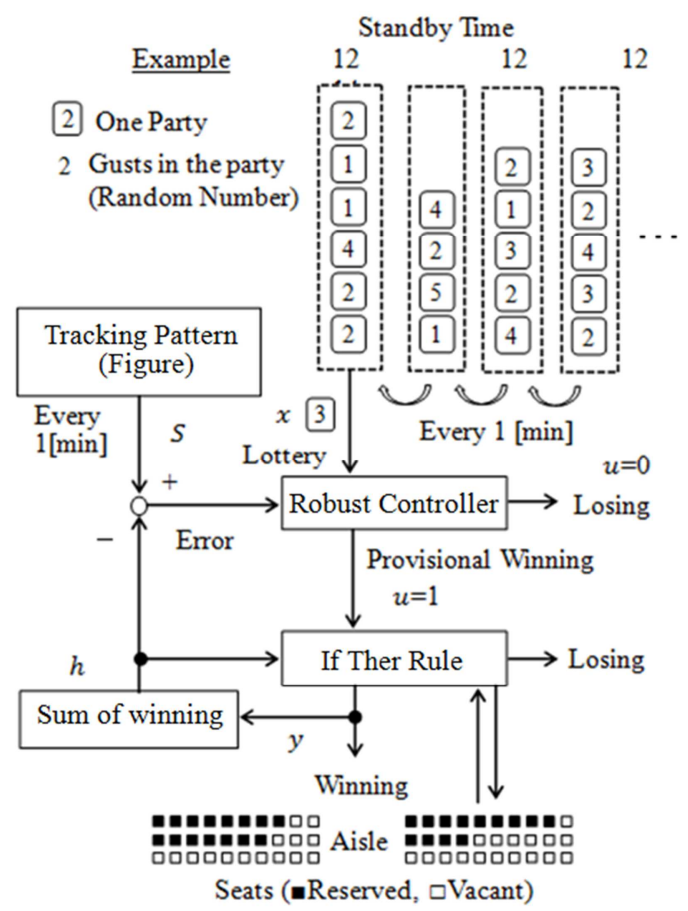

Figure 2. Simulation System (The value in the figure is an example for explanation).

Table 1. Nomenclature.

\begin{tabular}{ll}
\hline Symbol & Remarks \\
\hline$t$ & Simulation time [min] $(0,1,2,3, \cdots)$ \\
$k$ & Control step $(1,2,3, \cdots)$ \\
$S_{t}$ & Target value (Figure 5): The total number of seats that should be \\
$x_{k}$ & reserved at a simulation time $t$ \\
$u_{k}$ & The number of guests in a party \\
$y_{k}$ & Manipulated variable: $=1$ Winning, $=0$ Losing \\
$h_{k}$ & Output of 'If Then Rule': $=x_{k} u_{k}$ \\
\hline
\end{tabular}

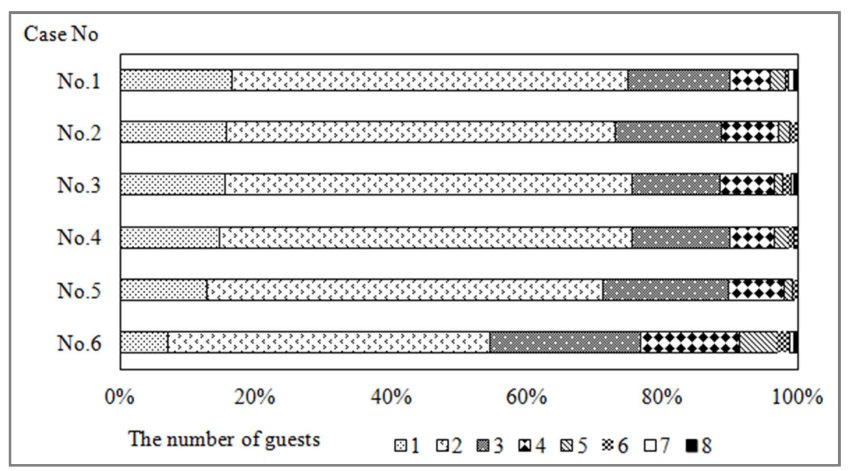

Figure 3. The number of guests in parties: Measurement (1).

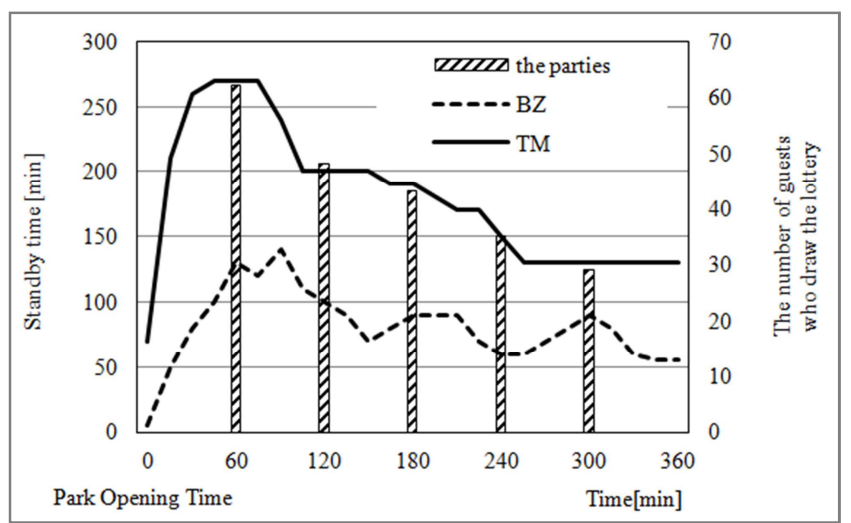

Figure 4. The time-series variations of guests who draw the lottery: Measurement (2) and time-series variations of the standby time (Example BZ: Buzz Lightyear's, TM: Toy Story Mania!).

\subsection{Input Data}

The number of parties, the number of people in a party, and the time when they draw the lottery are unknown. We guess these as follows;

Measurement (1):

To get the number of guests in a party $(x)$, we counted the number of guests of 3481 parties in total at the different date, time, and place ( 6 cases in total). Figure 3 shows the results.

Measurement (2):

To obtain the time-series variations of guests who draw the lottery, we counted the number of guests who come in the lottery place during 3700 minutes in total at a different date and time. As a results, we find that the time-series variations of Measurement (2) is equal to that of the standby time to enjoy the popular attraction which has a priority admission ticket (Fast-Pass ticket). Figure 4 shows the data of a certain one-day. Then we calculate the number of guests who draw the lottery in 1 minute using the standby time recorded every 15 minutes of the attractions. It becomes the input data of the simulation system.

Measurement (3):

We acquire the standby time of each attraction that the official website shows [9]. Details are as follows.

- Attractions: 12 (Buzz Lightyear's Astro Blasters, Big Thunder Mountain, Star Tours, Tower of Terror, Toy Story Mania!, and so on)

- Period: 01/Jan/2014 - 31/Dec/2014 
- Definition of one record: Standby time every 15 minutes of one day at one attraction

- The total number of records: 2658 (Disneyland 1501, DisneySea 1157. We removed the record having an abnormal data or lack data.)

The time-series variations of guests who draw the lottery in Tokyo Disney Park have a variety of choices, for example, there are many guests who come in the evening. These are reproduced by the records of 12 attractions of 365 days.

Process (1): (every 15[min] $\rightarrow$ every $1[\mathrm{~min}])$

We divide standby time data of every 15 minutes by 15 in straight line interpolation, and obtain the data every 1 minute.

Process (2): (1[min] $\rightarrow$ guests)

We assume the maximum of the number of guests in a party to be 8 (Refer Figure 3) and partition off the value of Process (1) by using a random number between 1 and 8 . The division result is the number of parties, and the value of the random number is the number of guests in a party (Refer Figure 2). We do not make the probability distribution of random number $(1-8)$ with a normal distribution, it is based on the composition ratio of Measurement (1), and we give the random vibration of 20 [\%] (based on Figure 3) as uncertainty of the composition ratio.

\subsection{Tracking Pattern}

We set the pre-desired tracking pattern [10] (See Figure 5) as a target value to meet Requirement (1) and (2). The abscissa of Figure 5 is the simulation time $(t)$, and the ordinate is the total number of seats $(S)$ that should be reversed at a simulation time. The total seating capacity of "Once Upon a Time" is 2500 .

\subsection{Robust Controller}

We apply ON/OFF-Type Discrete Variable Structure Controller $[6,7,8]$ because of the following reasons;

- To follow the tracking pattern (Refer Figure 5), the control logic must be a feedback controller.

- The input data of the simulation have the uncertainties. Therefore, the control logic must have robustness.

- The manipulated variable takes two values of Winning $(\mathrm{ON}: u=1)$ or Losing (OFF: $u=0)$.

- The simulation model is a discrete time.

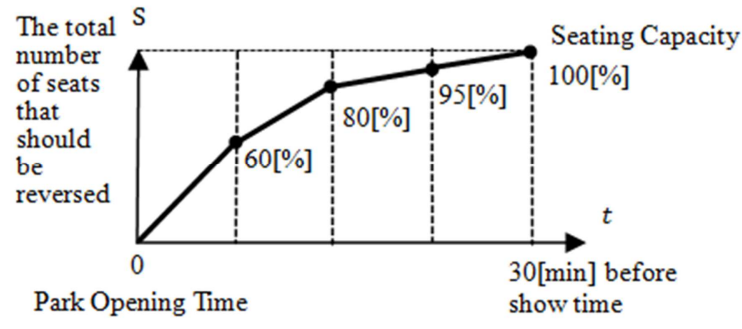

Figure 5. Tracking Pattern.

We derive the control logic satisfying Discrete Variable Structure theory condition to guarantee that an error decreases.

First, we define the error as follows,

$$
e_{k} \triangleq S_{t}-h_{k}
$$

At the next control step, there is the case which the target value changes (See Figure 6).

$$
\left\{\begin{array}{c}
e_{k+1}=S_{t}-h_{k+1} \\
e_{k+1}=S_{t+1}-h_{k+1}
\end{array}\right.
$$

The total of the reserved seats is calculated,

$$
h_{k+1}=h_{k}+y_{k}
$$

Next, we define the increment seats of the target value

$$
\Delta S \triangleq S_{t+1}-S_{t}(\Delta S \geq 0)
$$

Substituting Eq. (1), Eq. (3) and Eq. (4) into Eq. (2) leads to

$$
\begin{aligned}
& e_{k+1}=S_{t}+\Delta S-h_{k+1} \\
= & S_{t}+\Delta S-h_{k}-x_{k} u_{k} \\
= & e_{k}+\Delta S-x_{k} u_{k}(\Delta S \geq 0)
\end{aligned}
$$

If Discrete Variable Structure theory condition;

$$
\begin{gathered}
\text { When } e_{k}>0 \text {, must be } e_{k+1}<e_{k} \\
\text { When } e_{k} \leq 0 \text {, must be } e_{k+1} \geq 0
\end{gathered}
$$

is satisfied, the error decrease. Find the condition of $u_{k}=1$ (Winning) to satisfy Eq. (6) and Eq. (7).

About Eq. (6):

$$
e_{k}-e_{k+1}>0
$$

Substituting Eq. (5) into Eq. (8) leads to

$$
\begin{gathered}
e_{k}-e_{k}-\Delta S+x_{k} u_{k}>0 \\
x_{k} u_{k}>\Delta S
\end{gathered}
$$$$
\text { As } \Delta S \geq 0, \therefore u_{k}=1 \text { when } e_{k}>0
$$

About Eq. (7):

$$
e_{k+1}=e_{k}+\Delta S-x_{k} u_{k} \geq 0
$$

To satisfy Eq. (10),

$$
\begin{gathered}
x_{k} u_{k} \leq e_{k}+\Delta S \\
\therefore u_{k}=1 \text { when } x_{k} \leq e_{k}+\Delta S \\
\left(x_{k}>0, e_{k} \leq 0, \Delta S \geq 0\right)
\end{gathered}
$$

Except Eq. (9) and Eq. (11), $u_{k}=0$.

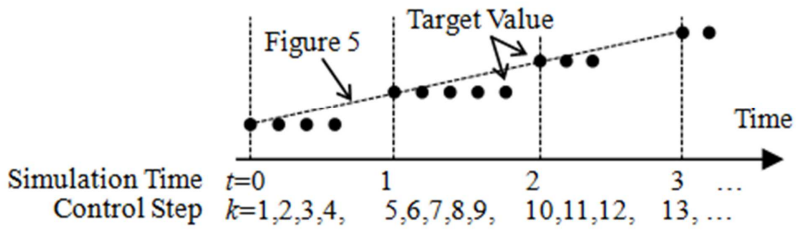

Figure 6. The details of the target value (The value in the figure is an example for explanation). 


\subsection{If Then Rule}

However, even if $u_{k}=1$, when any of the following condition is satisfied, change $u_{k}=0$. We call this 'If Then Rule' (Refer Figure 2).

- To solve Problem (1), when $u_{k}=1$ (Winning) has chosen continuously, if The number of consecutive winning $\geq$ Random number between 3 and 5 , then $u_{k}=0$. The random number is generated whenever the control logic operates.

- To meet Requirement (3), if this system cannot reserve the seats without sandwiching the aisle between guests in the winner party, then $u_{k}=0$.

\section{Simulation Result}

Table 2 shows the simulation result. Among simulation data of 2658 records (Refer Measurement (3)), we obtain an effective result that the each vacant seats of 2598 records are less than 50 seats ( 50 is $2[\%]$ of the seating capacity 2500 ). On the other hand, we obtain 25 records that the vacant seats are greater than or equal to 1000 seats.

Figure 7 and Figure 8 show one case of the simulation result. Because there are a few guests who draw the lottery at the park opening time (many guests get Fast-Pass ticket at first), the error is big at an early time (Refer Figure 7). And then it shows the good followability to the tracking pattern. Figure 8 shows that 'If Then Rule' can prevent the continuance of winning.

Table 2. Simulation Result

\begin{tabular}{llllll}
\hline Vacant seats & $\mathbf{0 - 4 9}$ & $\mathbf{5 0 - 9 9}$ & $\mathbf{1 0 0 - 9 9 9}$ & $\mathbf{1 0 0 0 -}$ & Total \\
\hline Records & 2598 & 12 & 23 & 25 & 2658 \\
\hline
\end{tabular}

\section{Neural Network Model}

In this chapter, we predict the case that the vacant seats increase by using NN (Neural Network Model) [11, 12].

About the bad 25 records in the simulation of Chap.3, which has many vacant seats the obvious reasons for the increase of vacant seats are as follows; foul weather, uncertain weather, or rain is forecasted. However, we cannot say these reasons apply for all bad records. It is difficult to clarify why the vacant seats increases or decreases.

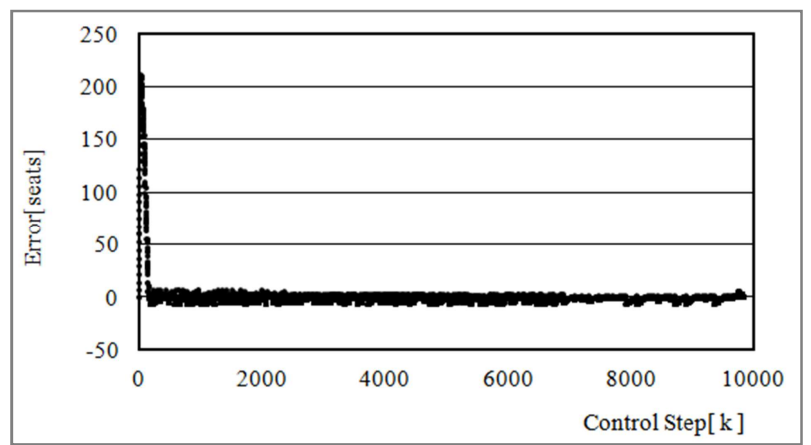

Figure 7. Error (Example, 01/Aug/2014, Attraction: Journey to the Center of the Earth).

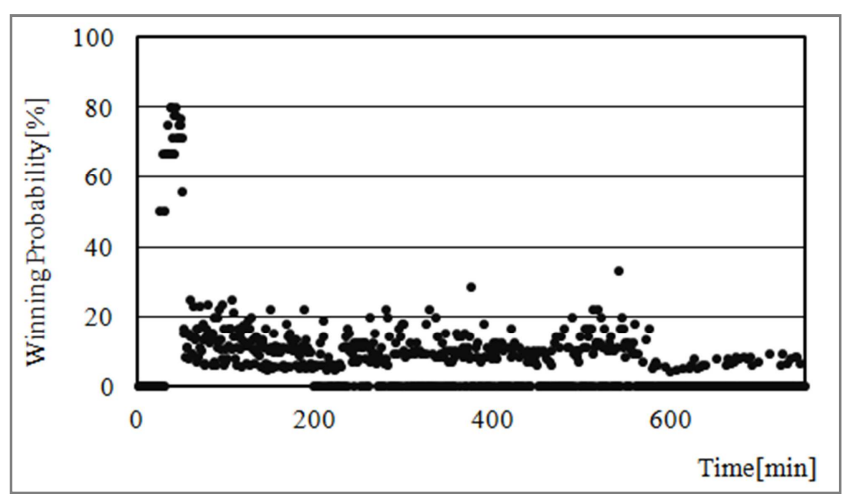

Figure 8. Winning Probability (Example, 01/Aug/2014, Attraction: Journey to the Center of the Earth).

To reduce the vacant seats, we propose that NN predicts the number of vacant seats at the end time of the lottery by using only the morning data. If the bad result is predicted, the staff of the lottery system is able to take the effective measures. For example, they can adjust the tracking pattern (Refer Figure 5) to increase the winners in the early afternoon.

$\mathrm{NN}$ has a conventional structure of the layers (input layer, hidden layer, output layer), and the learning method of $\mathrm{NN}$ is performed by a conventional error back-propagation algorithm. We calculate NN using R ( $\mathrm{R}$ is a free software environment for statistical computing and graphics) [13].

Construction:

Input data $=$ the morning data (from Park opening until noon) in the record (2658, 01/Jan/2014 - 31/Dec/2014).

Output data $=$ the number of vacant seats.

Learning data;

Model error $=$ Simulation result of Chap. 3 - Output data.

Effect:

Input data $=$ the morning data (from Park opening until noon) in New records (644, 01/Jan/2015 - 30/Apr/2015).

Output data $=$ the number of vacant seats.

Results; Table 3(A), (B)

Table 3(A). Simulation Result (New Records).

\begin{tabular}{llllll}
\hline Vacant Seats & $\mathbf{0 - 4 9}$ & $\mathbf{5 0 - 9 9}$ & $\mathbf{1 0 0 - 9 9 9}$ & $\mathbf{1 0 0 0}-$ & Total \\
\hline Records $^{* 1}$ & 627 & 6 & 5 & $6^{* 2}$ & 644 \\
\hline
\end{tabular}

*1 Simulation method same as Chap. II and Chap. III

${ }^{* 2}$ Refer Table 3(B): 6 cases

Table 3(B). Simulation Result (Vacant Seats: 1000-).

\begin{tabular}{|c|c|c|c|c|c|c|}
\hline Simulation Result $* 3$ & 1229 & 1781 & 1850 & 2047 & 2142 & 2176 \\
\hline NN Output*4 & 1250 & null & 1058 & null & 2128 & null \\
\hline
\end{tabular}

In this simulation, including null ( $\mathrm{NN}$ did not output a solution), NN succeeds in predicting the bad $6^{* 2}$ records which the vacant seats are greater than or equal to 1000 (Refer Table $3(\mathrm{~B})$ ). This result shows the effectiveness of NN. However, as 13 cases that the vacant seats of the simulation result are less 
than $50, \mathrm{NN}$ predicts that the each vacant seat is more than 1000. As the reason, we think that there are many guests of entering in the evening of the day, or weather is restored in the afternoon, and so on. NN can stand further improvement, which is a future task.

\section{Conclusion}

We applied the robust feedback controller, ON/OFF-Type Discrete Variable Structure Controller, for the lottery system in Tokyo Disney Park. The main features are as follows:

- This system realizes fine access control characterizing the feedback control to meet Requirement (1)-(3).

- The control logic solves Problem (2) because it does not use the database, and 'IF Then Rule' solves Problem (1).

- Simulation results, based on many real data more than one year and the random vibration of $20[\%]$ as uncertainty, show the effectiveness.

- NN predicts the number of vacant seats by using only the morning data. This method is useful to solve Problem (2).

In this paper, we have applied the new lottery system to one show ("Once Upon a Time"), but have confirmed that it is applicable to another shows ("Big Band Beat" and/or "One Man's Dream II"). Furthermore, if a theme park other than Tokyo Disney Park introduces a lottery system, this proposed system is applicable.

\section{References}

[1] Tokyo Disney Resort Official Website; http://www.tokyodisneyresort.jp/en/ (at 10/Mar/2015).

[2] XING Yuhong, "The Research and Design of an Applied Electronic Lottery System”, Lect Notes Comput Sci, Vol. 7719, pp.653-658, 2013.

[3] William Zame, Jie Xu and Mihaela van der Schaar, "Winning the Lottery: Learning Perfect Coordination With Minimal
Feedback", IEEE Journal of Selected Topics in Signal Processing, Vol. 7, No.5, pp. 846-857, 2013.

[4] YANG Jun, LIU Ying, QIN Ping, LIU Antung A, “A review of Beijing's vehicle registration lottery: Short-term effects on vehicle growth and fuel consumption", Energy Policy, Vol. 75 pp. 157-166, 2014.

[5] Title of Invention, "LOTTERY SYSTEM AND LOTTERY INFORMATION PROCESSOR", the name of the invention: Oriental Land Co., Ltd, The publication number assigned to the patent application by the patent office: 2005-266842 (in Japanese).

[6] T. Iwata, M. Yamakita and K. Furuta, "PWM-Type Discrete VSS Controller for On/Off Actuator Systems", Proc. Of the 39th IEEE Conference on Decision and Control, Sydney, Australia, Dec., pp.5131-5136, 2000.

[7] Msc Oscar Ivan Higuera Martinez, Juan Mauricio Salamanca and Hernando Diaz Morales, "Discrete-Time Variable Structure Control for Switching Converters", Transmission and Distribution Conference Conference and Exposition, IEEE/PES, Date 13-15 Aug, 2008

[8] Magnus Hedlund, Janaina G. Oliveira, Hans Bernhoff, "Sliding mode 4-quadrant DCDC converter for a fywheel application", Control Engineering Practice, Vol.21, pp.473-482, 2013.

[9] http://www.deepdisney.com/kimagure2012.aspx, http://s.tokyodisneyresort.jp/tdl/atrc_list.htm, http://s.tokyodisneyresort.jp/tds/atrc_list.htm(at 10/Mar/2015).

[10] N. Sakamoto, "The temperature control of the dryness fireplace by the sliding mode control", SICE Annual Conference 2002, WA10-3, pp.5-7.

[11] Yu-Wei. Chiu, "Machine Learning With R Cookbook - 110 Recipes for Building Powerful Predictive Models with R", Packt Publishing - ebooks Account, March 31, 2015.

[12] Brett Lantz, "Machine Learning with R - Second Edition", Packt Publishing - ebooks Account, Aug 3, 2015.

[13] R: The $\mathrm{R}$ Project for Statistical Computing Website; http://www.r-project.org/NeuralNetworkswithR;cran.r-project. org/web/packages/nnet/nnet.pdf (at 10/Mar/2015). 